\title{
AN INTERPOLATION INEQUALITY INVOLVING HÖLDER NORMS
}

\author{
ALOIS KUFNER AND ANDREAS WANNEBO
}

\begin{abstract}
An interpolation inequality of Nirenberg, involving Lebesgue-space norms of functions and their derivatives, is modified, replacing one of the norms by a Hölder norm.
\end{abstract}

\section{INTRODUCTION}

In his paper [1], L. Nirenberg derived the inequality

$$
\left\|\nabla^{j} u\right\|_{q} \leqq C\left\|\nabla^{m} u\right\|_{p}^{a}\|u\|_{r}^{1-a}
$$

which holds for all functions $u \in C_{0}^{\infty}\left(\mathbb{R}^{N}\right)$ with a constant $C>0$ independent of $u$. Here $\|\cdot\|_{s}$ is the $L^{s}$-norm, $\nabla^{k} u$ is the vector of all derivatives $D^{\alpha} u$ of order $|\alpha|=k, k \in \mathbb{N}$, and the parameters $p, q, r$ are connected, for $0<a<1$ and $0<j<m$, by the "dilation formula"

$$
-j+\frac{N}{q}=a\left(-m+\frac{N}{p}\right)+(1-a) \frac{N}{r} .
$$

Moreover, it is shown that the parameter $a$ has to satisfy the condition

$$
a \geqq \frac{j}{m}
$$

Inequality (0.1) was, among others, a very important tool in the description of properties of Sobolev spaces $W^{m, p}\left(\mathbb{R}^{n}\right)$. For example, for the limiting cases $j=0$ and $a=1$, we obtain from (0.1) the famous Sobolev Imbedding theorem

$$
\|u\|_{q} \leqq C\left\|\nabla^{m} u\right\|_{p} \quad \text { with } \quad \frac{1}{q}=\frac{1}{p}-\frac{m}{N} .
$$

1991 Mathematics Subject Classification. 26D10, 46E35.

Key words and phrases. Interpolation inequalities, Hölder norms, Sobolev imbedding, dilation formula.

603

1072-947X/95/1100-0603\$07.50/0 (c) 1995 Plenum Publishing Corporation 
The aim of this note is to modify inequality $(0.1)$ replacing the $L^{r}$-norm of $u,\|u\|_{r}$ on the right-hand side by the Hölder quotient

$$
[u]_{H(\lambda)}=\sup _{x \neq y} \frac{|u(x)-u(y)|}{|x-y|^{\lambda}}, \quad 0<\lambda<1
$$

i.e., to derive inequalities of the form

$$
\left\|\nabla^{j} u\right\|_{q} \leqq C\left\|\nabla^{m} u\right\|_{p}^{a}[u]_{H(\lambda)}^{1-a}
$$

for appropriate values of the parameters $j, m, p, q, \lambda, a$.

First, let us note that the formula

$$
-j+\frac{N}{q}=a\left(-m+\frac{N}{p}\right)+(1-a)(-\lambda)
$$

is an analogue of formula (0.2) for the case of inequality (0.4). Indeed, if (0.4) holds for every function $u=u(x) \in C_{0}^{\infty}\left(\mathbb{R}^{n}\right)$ with a constant $C>0$ independent of $u$, then it holds necessarily for the function $U(x)=u(R x)$ with $R>0$, which again belongs to $C_{0}^{\infty}\left(\mathbb{R}^{n}\right)$. From $(0.4)$ we obtain that

$$
\left\|\nabla^{j} U\right\|_{q} R^{-j+\frac{N}{p}} \leqq C\left\|\nabla^{m} U\right\|_{p}^{a} R^{a\left(-m+\frac{N}{p}\right)}[u]_{H(\lambda)}^{1-a} R^{-\lambda(1-a)}
$$

and (0.5) follows since $R>0$ is arbitrary.

The paper is organized as follows: in Section 1, we will derive an important auxiliary estimate (Lemma 1). In Section 2, we will first deal with inequality (0.4) for the one-dimensional case (Theorem 1) and then, in Section 3 , the result will be extended to functions defined on $\mathbb{R}^{N}, N>1$, but under certain more restrictive conditions on the parameters (Theorem 2).

\section{An AUXiliary RESUlt}

Lemma 1. Let $u=u(t)$ be a smooth function on the finite closed interval $I \subset \mathbb{R}$. Suppose $m, j \in \mathbb{N}, 0<j<m, 0<\lambda \leqq 1$ and denote

$$
[u]_{\lambda, I}=\sup \left\{\frac{|u(t)-u(s)|}{|t-s|^{\lambda}} ; t, s \in I, t \neq s\right\} .
$$

Then the estimate

$$
\left|u^{(j)}(t)\right| \leqq K\left\{|I|^{m-j-1} \int_{I}\left|u^{(m)}(s)\right| d s+|I|^{\lambda-j}[u]_{\lambda, I}\right\}
$$

holds for every $t \in I$ with $K>0$ independent of $u, t$ and the length $|I|$ of the interval $I: K=K(j, m, \lambda)$. 
Proof. Without loss of generality, we can assume that $I=[0, b], 0<b<\infty$.

(i) Take $\xi \in\left[0, \frac{1}{3} b\right], \eta \in\left[\frac{2}{3} b, b\right]$. Then there is an $x \in[\xi, \eta]$ such that

$$
u(\xi)-u(\eta)=u^{\prime}(x)(\xi-\eta),
$$

i.e.,

$$
\left|u^{\prime}(x)\right|=\frac{|u(\xi)-u(\eta)|}{|\xi-\eta|}=\frac{|u(\xi)-u(\eta)|}{|\xi-\eta|^{\lambda}}|\xi-\eta|^{\lambda-1},
$$

and since $|\xi-\eta| \geqq \frac{1}{3} b$ and $\lambda-1 \leqq 0$, we have

$$
\left|u^{\prime}(x)\right| \leqq[u]_{\lambda, I}\left(\frac{b}{3}\right)^{\lambda-1}
$$

Let us fix this $x$ and take any $t \in[0, b]$. Then

$$
u^{\prime}(t)=\int_{x}^{t} u^{\prime \prime}(s) d s+u^{\prime}(x)
$$

and consequently

$$
\left|u^{\prime}(t)\right| \leqq \int_{0}^{b}\left|u^{\prime \prime}(s)\right| d s+\left|u^{\prime}(x)\right| \leqq \int_{0}^{b}\left|u^{\prime \prime}(s)\right| d s+3^{1-\lambda} b^{\lambda-1}[u]_{\lambda, I}
$$

due to (1.2). But (1.3) is (1.1) for $j=1, m=2$.

(ii) Take $\xi_{0} \in\left[0, \frac{1}{9} b\right], \xi_{1} \in\left[\frac{2}{9} b, \frac{1}{3} b\right]$. Then there is a $\xi \in\left[\xi_{0}, \xi_{1}\right]-$ i.e., $\xi \in\left[0, \frac{1}{3} b\right]-$ such that

$$
u\left(\xi_{0}\right)-u\left(\xi_{1}\right)=u^{\prime}(\xi)\left(\xi_{0}-\xi_{1}\right) .
$$

Further, take $\eta_{0} \in\left[\frac{2}{3} b, \frac{7}{9} b\right], \eta_{1} \in\left[\frac{8}{9} b, b\right]$. Then there is an $\eta \in\left[\eta_{0}, \eta_{1}\right]$ - i.e., $\eta \in\left[\frac{2}{3} b, b\right]-$ such that

$$
u\left(\eta_{0}\right)-u\left(\eta_{1}\right)=u^{\prime}(\eta)\left(\eta_{0}-\eta_{1}\right) .
$$

Moreover, there is an $x \in[\xi, \eta]$ such that

$$
u^{\prime}(\xi)-u^{\prime}(\eta)=u^{\prime \prime}(x)(\xi-\eta) .
$$

Consequently,

$$
u^{\prime \prime}(x)=\frac{u^{\prime}(\xi)-u^{\prime}(\eta)}{\xi-\eta}=\frac{1}{\xi-\eta}\left[\frac{u\left(\xi_{0}\right)-u\left(\xi_{1}\right)}{\xi_{0}-\xi_{1}}-\frac{u\left(\eta_{0}\right)-u\left(\eta_{1}\right)}{\eta_{0}-\eta_{1}}\right],
$$

and since $|\xi-\eta| \geqq \frac{1}{3} b,\left|\xi_{0}-\xi_{1}\right| \geqq \frac{1}{9} b,\left|\eta_{0}-\eta_{1}\right| \geqq \frac{1}{9} b$, we have

$$
\begin{gathered}
\left|u^{\prime \prime}(x)\right| \leqq \\
\frac{1}{|\xi-\eta|}\left[\frac{\left|u\left(\xi_{0}\right)-u\left(\xi_{1}\right)\right|}{\left|\xi_{0}-\xi_{1}\right|^{\lambda}}\left|\xi_{0}-\xi_{1}\right|^{\lambda-1}+\frac{\left|u\left(\eta_{0}\right)-u\left(\eta_{1}\right)\right|}{\left|\eta_{0}-\eta_{1}\right|^{\lambda}}\left|\eta_{0}-\eta_{1}\right|^{\lambda-1}\right] \leqq
\end{gathered}
$$




$$
\leqq \frac{3}{b} 2[u]_{\lambda, I}\left(\frac{b}{9}\right)^{\lambda-1}=6 \cdot 9^{1-\lambda} b^{\lambda-2}[u]_{\lambda, I} .
$$

Let us fix this $x$ and take any $t \in[0, b]$. Then

$$
u^{\prime \prime}(t)=\int_{x}^{t} u^{\prime \prime \prime}(s) d s+u^{\prime \prime}(x)
$$

and consequently, due to (1.4)

$$
\left|u^{\prime \prime}(t)\right| \leqq \int_{0}^{b}\left|u^{\prime \prime \prime}(s)\right| d s+6 \cdot 9^{1-\lambda} b^{\lambda-2}[u]_{\lambda, I} .
$$

But this is (1.1) for $j=2, m=3$.

(iii) Integrating (1.5) with respect to $t$ over the interval $[0, b]$, we obtain that

$$
\begin{gathered}
\int_{0}^{b}\left|u^{\prime \prime}(t)\right| d t \leqq b\left[\int_{0}^{b}\left|u^{\prime \prime \prime}(s)\right| d s+6 \cdot 9^{1-\lambda} b^{\lambda-2}[u]_{\lambda, I}\right]= \\
=b \int_{0}^{b}\left|u^{\prime \prime \prime}(s)\right| d s+6 \cdot 9^{1-\lambda} b^{\lambda-1}[u]_{\lambda, I} .
\end{gathered}
$$

Using this estimate in (1.3), we see that

$$
\begin{gathered}
\left|u^{\prime}(t)\right| \leqq b \int_{0}^{b}\left|u^{\prime \prime \prime}(s)\right| d s+6 \cdot 9^{1-\lambda} b^{\lambda-1}[u]_{\lambda}+3^{1-\lambda} b^{\lambda-1}[u]_{\lambda, I}= \\
=b \int_{0}^{b}\left|u^{\prime \prime \prime}(s)\right| d s+K b^{\lambda-1}[u]_{\lambda, I}
\end{gathered}
$$

with $K=6 \cdot 9^{1-\lambda}+3^{1-\lambda}$. But this is $(1.1)$ for $j=1, m=3$.

(iv) The proof for general $j, m \in \mathbb{N}(j<m)$ proceeds by induction. First, we show that there is an $x \in[0, b]$ such that

$$
\left|u^{(j)}(x)\right| \leqq K(j)[u]_{\lambda, I} b^{\lambda-j}
$$

with $K(j)=2^{j-1} 3^{\frac{j}{2}(j-2 \lambda+1)}$ [compare with (1.2) and (1.4) for $j=1$ and $j=2$, respectively]. 
Putting this $x$ fixed and taking any $x \in[0, b]$, we obtain from

$$
u^{(j)}(t)=\int_{x}^{t} u^{(j+1)}(s) d s+u^{(j)}(x)
$$

that

$$
\left|u^{(j)}(t)\right| \leqq \int_{0}^{b}\left|u^{(j+1)}(s)\right| d s+K(j) b^{\lambda-j}[u]_{\lambda, I}
$$

and integration with respect to $t$ over $[0, b]$ yields

$$
\int_{0}^{b}\left|u^{(j)}(t)\right| \leqq b \int_{0}^{b}\left|u^{(j+1)}(s)\right| d s+K(j) b^{\lambda-j+1}[u]_{\lambda, I} .
$$

For $j=m-1,(1.6)$ is the estimate (1.1).

For $j=m-2$, estimate (1.6) yields

$$
\left|u^{(m-2)}(t)\right| \leqq \int_{0}^{b}\left|u^{(m-1)}(s)\right| d s+K(m-2) b^{\lambda-m+2}[u]_{\lambda, I}
$$

while (1.7) yields, for $j=m-1$, that

$$
\int_{0}^{b}\left|u^{(m-1)}(s)\right| d s \leqq b \int_{0}^{b}\left|u^{(m)}(s)\right| d s+K(m-1) b^{\lambda-m+2}[u]_{\lambda, I} .
$$

Using this estimate in (1.8), we immediately obtain (1.1) for $j=m-2$ with $K=K(m-1)+K(m-2)$.

Analogously we proceed for $j=m-3, m-4, \ldots$.

Remark. Inequality (1.1) is a counterpart of the inequality

$$
\left|u^{(j)}(t)\right| \leqq K\left\{\left.|I|^{m-j-1} \int_{I}\left|u^{(m)}(s) d s+\right| I\right|^{-j-1} \int_{I}|u(s)| d s\right\}
$$

which is a useful tool when deriving interpolation inequalities in (weighted) $L^{s}$-norms (see, e.g., R.C. Brown and D.B. Hinton [2]).

Suppose $1<p, q<\infty$. Then we can immediately derive from Lemma 1 the following 
Corollary. Under the assumptions of Lemma 1, the estimate

$$
\begin{gathered}
\int_{I}\left|u^{(j)}(t)\right|^{q} d t \leqq \\
\leqq \widetilde{K}\left\{|I|^{(m-j) q+1-\frac{q}{p}}\left(\int_{I}\left|u^{(m)}(s)\right|^{p} d s\right)^{q / p}+|I|^{1+(\lambda-j) q}[u]_{\lambda, I}^{q}\right\}
\end{gathered}
$$

holds.

Proof. The Hölder inequality yields for $1<p<\infty$ that

$$
\int_{I}\left|u^{(m)}(s)\right| d s \leqq\left(\int_{I}\left|u^{(m)}(s)\right|^{p} d s\right)^{1 / p}|I|^{1-\frac{1}{p}} .
$$

For $1<q<\infty$, if follows from (1.1) that

$$
\begin{gathered}
\left|u^{(j)}(t)\right|^{q} \leqq \\
\leqq 2^{q-1} K\left\{|I|^{(m-j-1) q}\left(\int_{I}\left|u^{(m)}(s)\right| d s\right)^{q}+|I|^{(\lambda-j) q}[u]_{\lambda, I}^{q}\right\}
\end{gathered}
$$

holds for every $t \in I$. Integrating this inequality with respect to $t$ over $I$ and using (1.10), we obtain the estimate (1.9).

\section{The OnE-Dimensional CASE}

Let us assume that $u=u(t)$ is defined on $\mathbb{R}_{+}$, that $0<j<\infty$, and that $u^{(m)} \in L^{p}\left(\mathbb{R}_{+}\right), u^{(j)} \in L^{q}\left(\mathbb{R}_{+}\right)$, and $[u]_{\lambda, \mathbb{R}_{+}}$is finite.

Consider first the interval $[0, L], 0<L<\infty$. Following the idea of L. Nirenberg [2], we will cover this interval by a finite number of successive intervals $I_{1}, I_{2}, \ldots$ where the initial point of $I_{i+1}$ coincides with the endpoint of $I_{i}$.

Take a fixed $k \in \mathbb{N}$ and consider the estimate (1.9) for the special interval $I=[0, L / k]$. If the first term on the right-hand side of (1.9) is greater than the second, then we set $I_{1}=I$ and hence we have the estimate

$$
\int_{I_{1}}\left|u^{(j)}(s)\right|^{q} d s \leqq 2 \widetilde{K}\left(\frac{L}{k}\right)^{\left(m-j-\frac{1}{p}\right) q+1}\left(\int_{I_{1}}\left|u^{(m)}(s)\right|^{p} d s\right)^{q / p} .
$$

On the other hand, if the second term is greater, we proceed in the following way: We suppose that

$$
1+(\lambda-j) q<0
$$


[in fact, this means that we have to suppose $\lambda<1-1 / q$ if $j=1$, since for $j=2,3, \ldots$ the condition (2.2) is satisfied due to the assumption $0<\lambda \leqq 1$, while

$$
\left(m-j-\frac{1}{p}\right) q+1>0
$$

and we introduce a parameter $a, 0<a<1$.

Now we extend the interval $I$ (keeping the left endpoint fixed) until the $a$-multiple of the second term becomes equal to the $(1-a)$-multiple of the first term. This must occur for a finite value of $|I|$, since the exponent on $|I|$ in the first term is positive due to (2.3), but the exponent on $|I|$ is negative due to (2.2). Denoting $I_{1}$ the resulting interval and using the identity

$$
A+B=\left(\frac{1}{a}\right)^{a}\left(\frac{1}{1-a}\right)^{1-a} A^{a} B^{1-a} \text { if } a B=(1-a) A,
$$

we then have

$$
\begin{gathered}
\int_{I_{1}}\left|u^{(j)}(s)\right|^{q} d s \leqq \widetilde{K}\left(\frac{1}{a}\right)^{a}\left(\frac{1}{1-a}\right)^{1-a}\left|I_{1}\right|^{\left(m-j-\frac{1}{p}\right) q a+a} \times \\
\times\left(\int_{I_{1}}\left|u^{(m)}(t)\right|^{p} d t\right)^{a q / p} \cdot\left|I_{1}\right|^{(1-a)(1+\lambda q-j q)}[u]_{\lambda, I_{1}}^{q(1-a)} .
\end{gathered}
$$

If we choose

$$
a=\frac{j-\frac{1}{q}-\lambda}{m-\frac{1}{p}-\lambda}
$$

then the foregoing estimate becomes simple:

$$
\int_{I_{1}}\left|u^{(j)}(s)\right|^{q} d s \leqq \widetilde{K}_{a}\left(\int_{I_{1}}\left|u^{(m)}(s)\right|^{p} d s\right)^{a q / p} \cdot[u]_{\lambda, I_{1}}^{q(1-a)} .
$$

Keeping $k$ fixed, we now start at the endpoint of $I_{1}$ and repeat this process [beginning with an interval of length $L / k$, comparing the two terms on the right-hand side of the corresponding inequality (1.9), etc.] choosing $I_{2}, I_{3}, \ldots$ until the interval $[0, l]$ is covered. There are at most $k$ such intervals, and if we now sum up our estimates of

$$
\int_{I_{i}}\left|u^{(j)}(s)\right|^{q} d s
$$


which are of the form (2.1) or (2.5), we finally find that

$$
\begin{gathered}
\int_{0}^{L}\left|u^{(j)}(s)\right|^{q} d s \leqq \sum_{i} \int_{I_{i}}\left|u^{(j)}(s)\right|^{q} d s \leqq \\
\leqq k \cdot 2 \widetilde{K}\left(\frac{L}{k}\right)^{\left(m-j-\frac{1}{p}\right) q+1}\left(\int_{0}^{\infty}\left|u^{(m)}(s)\right|^{p} d s\right)^{q / p}+ \\
+\widetilde{K}_{a} \sum_{i}\left(\int_{I_{i}}\left|u^{(m)}(t)\right|^{p} d t\right)^{a q / p} \cdot[u]_{\lambda, I_{i}}^{q(1-a)} .
\end{gathered}
$$

If we suppose

$$
\frac{a q}{p} \geqq 1
$$

which in fact means that

$$
\lambda \leqq \frac{j q-m p}{q-p}
$$

and which contains the assumption $j q-m p>0$, i.e.,

$$
q>\frac{m}{j} p
$$

then

$$
\begin{aligned}
& \sum_{i}\left(\int_{I_{i}}\left|u^{(m)}(t)\right|^{p} d t\right)^{a q / p} \cdot[u]_{\lambda, I_{i}}^{q(1-a)} \leqq \\
& \leqq\left\{\sum_{i}\left(\int_{I_{i}}\left|u^{(m)}(t)\right|^{p} d t\right)^{a q / p}\right\} \cdot[u]_{\lambda, \mathbb{R}_{+}}^{q(1-a)} \leqq \\
& \leqq\left\{\sum_{i}\left(\int_{I_{i}}\left|u^{(m)}(t)\right|^{p} d t\right)\right\}^{a q / p} \cdot[u]_{\lambda, \mathbb{R}_{+}}^{q(1-a)} \leqq \\
& \leqq\left(\int_{0}^{\infty}\left|u^{(m)}(t)\right|^{p} d t\right)^{a q / p} \cdot[u]_{\lambda, \mathbb{R}_{+}}^{q(1-a)} .
\end{aligned}
$$

This is a (global) bound for the second term on the right-hand side of (2.6). If we now let $k \rightarrow \infty$, then the first term tends to zero, since $\left(m-j-\frac{1}{p}\right) q+$ $1>1$, and we obtain the interpolation inequality

$$
\left(\int_{0}^{\infty}\left|u^{(j)}(t)\right|^{q} d t\right)^{1 / q} \leqq C\left(\int_{0}^{\infty}\left|u^{(m)}(t)\right|^{p} d t\right)^{a / p} \cdot[u]_{\lambda, \mathbb{R}_{+}}^{1-a}
$$


since the number $L$ on the left-hand side of (2.6) was arbitrary.

Let us summarize the result.

Theorem 1. Suppose $m, j \in \mathbb{N}, 0<j<m, 1<p<q<\infty, 0<\lambda \leqq 1$, $0<\lambda<1-\frac{1}{q}$, if $j=1$. Further suppose that

$$
q>\frac{m}{j} p
$$

and

$$
\lambda \leqq \frac{j q-m p}{q-p}
$$

Then the interpolation inequality

$$
\left\|u^{(j)}\right\|_{q} \leqq C\left\|u^{(m)}\right\|_{p}^{a} \cdot[u]_{H(\lambda)}^{1-a}
$$

holds for every $u \in C_{0}^{\infty}\left(\mathbb{R}_{+}\right)$with

$$
a=\frac{j-\frac{1}{p}-\lambda}{m-\frac{1}{p}-\lambda} .
$$

\section{The $N$-Dimensional CASE}

Theorem 2. Suppose $N, m, j \in \mathbb{N}, N \geqq 2,0<j<m, 1<p<q<\infty$. Further, let

$$
\frac{m}{j} p<q \leqq \frac{m-1}{j-1} p
$$

and

$$
\lambda=\frac{j q-m p}{q-p} .
$$

Then the interpolation inequality (0.4),

$$
\left\|\nabla^{j} u\right\|_{q} \leqq C\left\|\nabla^{m} u\right\|_{p}^{a} \cdot[u]_{H(\lambda)}^{1-a},
$$

holds for every $u \in C_{0}^{\infty}\left(\mathbb{R}^{N}\right)$ with

$$
a=\frac{p}{q} .
$$

Proof. For $x \in \mathbb{R}^{N}$ denote $x=\left(t, x^{\prime}\right)$ with $t \in \mathbb{R}$ and $x^{\prime} \in \mathbb{R}^{N-1}$. For any fixed $x^{\prime}$ we can rewrite the inequality (2.11) [i.e., (2.10), but now on $\mathbb{R}$ instead of $\mathbb{R}_{+}$] in the form

$$
\int_{-\infty}^{+\infty}\left|\frac{\partial^{j} u}{\partial t^{j}}\left(x^{\prime}, t\right)\right|^{q} d t \leqq C^{q}\left(\int_{-\infty}^{+\infty}\left|\frac{\partial^{m} u}{\partial t^{m}}\left(x^{\prime}, t\right)\right|^{p} d t\right)^{a q / p} \cdot\left[u\left(x^{\prime}, \cdot\right)\right]_{\lambda, \mathbb{R}_{+}}^{(1-a) q} .
$$


Estimating $\left.[u]\left(x^{\prime}, \cdot\right)\right]_{\lambda, \mathbb{R}}$ by $[u]_{H(\lambda)}$ and integrating the resulting inequality with respect to $x^{\prime} \in \mathbb{R}^{N-1}$, we obtain that

$$
\begin{aligned}
\int_{\mathbb{R}^{N}}\left|\frac{\partial^{j} u}{\partial t^{j}}(x)\right|^{q} d x & \leqq C\left(\int_{\mathbb{R}^{N-1}}\left[\int_{\mathbb{R}}\left|\frac{\partial^{m} u}{\partial t^{m}}\left(x^{\prime}, t\right)\right|^{p} d t\right]^{a q / p} d x^{\prime}\right) \cdot[u]_{H(\lambda)}^{(1-a) q}= \\
& =C^{p}\left(\int_{\mathbb{R}^{N}}\left|\frac{\partial^{m} u}{\partial t^{m}}(x)\right|^{p} d x\right)^{a q / p} \cdot[u]_{H(\lambda)}^{(1-a) q}
\end{aligned}
$$

since due to (3.4), $a q / p=1$. Now (3.3) follows immediately, taking the $1 / q$ th power of both sides.

Due to (3.4), the "dilation formula" (0.5) has now the form

$$
-j+\frac{N}{q}=\frac{p}{q}\left(-m+\frac{N}{p}\right)+\frac{p-q}{q} \lambda
$$

which leads to formula (3.2), and since $0<\lambda \leqq 1$, we obtain the conditions (3.1).

\section{AKNOWLEDGMENT}

This paper was written while the authors visited each other within the framework of collaboration between the Academy of Sciences of the Czech Republic and the Swedish Royal Academy of Sciences. Morever, the first author was partially supported by the Grant Agency of the Czech Republic, project No. 201/94/1066, which is gratefully aknowledged.

\section{REFERENCES}

1. L. Nirenberg, On elliptic partial differential equations. Ann. Scuola Norm. Sup. Pisa 13(1959), 115-162.

2. R. C. Brown and D. B. Hinton, Sufficient conditions for weighted inequalities of sum form. J. Math. Anal. Appl. 112(1985), 563-578.

(Received 09.06.1994)

Authors' addresses:

Alois Kufner

Mathematical Institute,

Acad. Sci. of the Czech Republic

Žitn 25, 11567 Praha 1,

Czech Republic
Andreas Wannebo

Dept. of Mathematics, Royal Institute of Technology S-10044 Stockholm, Sweden 